\title{
Detection of Mycoplasma pneumoniae and Chlamydia pneumoniae in ruptured atherosclerotic plaques
}

M.L. Higuchi, N. Sambiase, S. Palomino, P. Gutierrez, L.M. Demarchi, V.D. Aiello and J.A.F. Ramires
Laboratório de Anatomia Patológica, Instituto do Coração,

Faculdade de Medicina, Universidade de São Paulo, São Paulo, SP, Brasil

\section{Correspondence \\ M.L. Higuchi \\ Laboratório de Anatomia Patológica \\ InCor, FM, USP \\ Av. Dr. Enéas $C$. Aguiar, 44 \\ 05403-000 São Paulo, SP \\ Brasil \\ Fax: + 55-11-3069-5251 \\ E-mail: anplourdes@ incor.usp.br}

Publication supported by FAPESP.

Received June 28, 2000

Accepted August 11, 2000

\begin{abstract}
This paper reports what is apparently the first observation of Mycoplasma pneumoniae in association with Chlamydia pneumoniae in thrombosed ruptured atheromas. We performed electron microscopy and in situ hybridization in specimens from three patients who died of acute myocardial infarction. These patients had typical symptoms of acute ischemic syndrome. Mycoplasmas were present mainly in the lipid core of the ruptured thrombosed plaque. Vulnerable atheromas are rich in cholesterol and may favor the growth of mycoplasmas, the only microorganisms that require cholesterol for survival. We suggest that the association of Mycoplasma pneumoniae and Chlamydia pneumoniae may increase the virulence of these microorganisms, favoring proliferation, plaque inflammation and possibly plaque rupture.
\end{abstract}

Key words

- Mycoplasma pneumoniae

- Chlamydia pneumoniae

- Atherosclerosis

- Vulnerable plaque

- Inflammation
It is currently accepted that atheroma inflammation is an important factor associated with plaque vulnerability (1). The role of Chlamydia pneumoniae in the development of atherosclerosis and plaque rupture is a controversial issue $(2,3)$. The difficulties are mainly related to the diversity of microorganisms found in atherosclerotic lesions. In order to clarify this matter, in a previous study we quantitatively estimated the amount of $C$. pneumoniae in different kinds of atherosclerotic coronary lesions and demonstrated larger numbers of this microorganism in vulnerable atheromatous plaques (4). However, the intensity of intraplaque inflammation did not correlated with the number of $C$. pneumoniae, suggesting that other factors may have a role in plaque instability and inflammation (Higuchi ML, Aiello VD,
Castelli JB, Palomino S, Reis MM, Sambiase N, Fukasawa S and Ramires JAS, unpublished data).

Presently, we report additional electron microscopic findings in specimens from 3 patients who died of acute myocardial infarction. These patients have been previously described as having a large number of C. pneumoniae bacteria in the ruptured thrombosed coronary artery responsible for their death (4). A more detailed analysis of the autopsy material demonstrated that another microorganism was present in the unstable segments in the intima in association with the $C$. pneumoniae bodies. The electron microscopic characteristics that allowed us to recognize them as Mycoplasma pneumoniae included rounded structures containing a granulous chromatin-like material enveloped 
by a cytoplasmic membrane, in the absence of an external bacterial wall. Mycoplasmas are the only bacteria that do not have an external wall (5). They also have a granulose amorphous material on their external surface. These bacteria were adhered to the endothelial surface of the vasa vasorum (Figure 1A) or were in the cytoplasm of cells also infected with $C$. pneumoniae. The mycoplasmas were present in blood monocytes and macrophages (Figure 1B) or in the interstitium. Large numbers of these microorganisms were present inside the atheroma together with $C$. pneumoniae and were associated with several membrane components possibly corresponding to degenerated bacteria (Figure 1C). We also found mycoplasmas in large cylindrical or elliptical forms in the extracellular matrix (Figure 1D). Our diagnosis of mycoplasma was confirmed by in situ hybridization with an M. pneumoniae-specific probe from Enzo Diagnostics (New York, NY, USA). This technique, which was described previously (6), revealed a larger number of mycoplasmas mainly in unstable plaque segments throughout the fatty material or in the necrotic core (Figure 1E and F).

Mycoplasmas are the smallest and simplest self-replicating microorganisms. $M y$ coplasma pneumoniae is usually known to cause upper and lower respiratory tract infections. However, many unanswered questions exist about their pathogenicity and involvement in disease. Macrophage activation, cytokine induction, and superantigen properties are some factors related to the pathogenicity of mycoplasmas (7). They are coccoid in culture but, depending on the nutritional medium, they may be elongated or filamentous (up to $100 \mu \mathrm{m}$ ). The most intriguing characteristic is that they require cholesterol for survival because their membrane is constituted of cholesterol, a unique property among prokaryotes. This may help to explain why larger numbers of microorganisms were found inside the lipid core of the atherosclerotic plaque. We may speculate that these organisms may induce macrophage activation, causing release of high levels of cytokines and perhaps apoptosis. Mycoplasma pneumoniae excretes hydrogen peroxide and superoxide radicals, thus possibly damaging the host cell membrane to which it closely adheres (7). The microorganisms are probably responsible for the oxidation of lipids in the plaque and for the death of macrophages. It is known that coinfections with other bacteria in association with M. pneumoniae potentiate the virulence of both infectious agents (8). Therefore, when both Mycoplasma pneumoniae and Chlamydia pneumoniae are present in atheromatous plaques they may increase each other's pathogenicity which in turn may lead to plaque rupture (9). Blasi et al. (10) did not find $M$. pneumoniae in atherosclerotic plaques of abdominal aortic aneurysms or of the carotid artery. In agreement with these findings, we observed that M. pneumoniae were mainly present in thrombosed ruptured plaques and less abundant in stable plaques. The association of Mycoplasma pneumoniae and Chlamydia pneumonia has been described in some case reports $(11,12)$, mainly causing pneumonia, or in immunodeficient patients. In atherosclerosis, an indirect serological finding has suggested a correlation between $M$. pneumoniae antibodies and coronary atherosclerosis (13). An experimental study (14) demonstrated that $M$. pneumoniae alone did not produce changes in rabbit atherosclerosis, favoring our hypothesis that the presence of both bacteria may be important for the development of plaque instability.

To the best of our knowledge, this is the first report showing M. pneumoniae in association with $C$. pneumoniae in atherosclerotic plaque. Investigating of the mechanisms by which $M$. pneumoniae and other microorganisms are involved in atheroma may lead to a better understanding of the pathogenesis of plaque instability. 

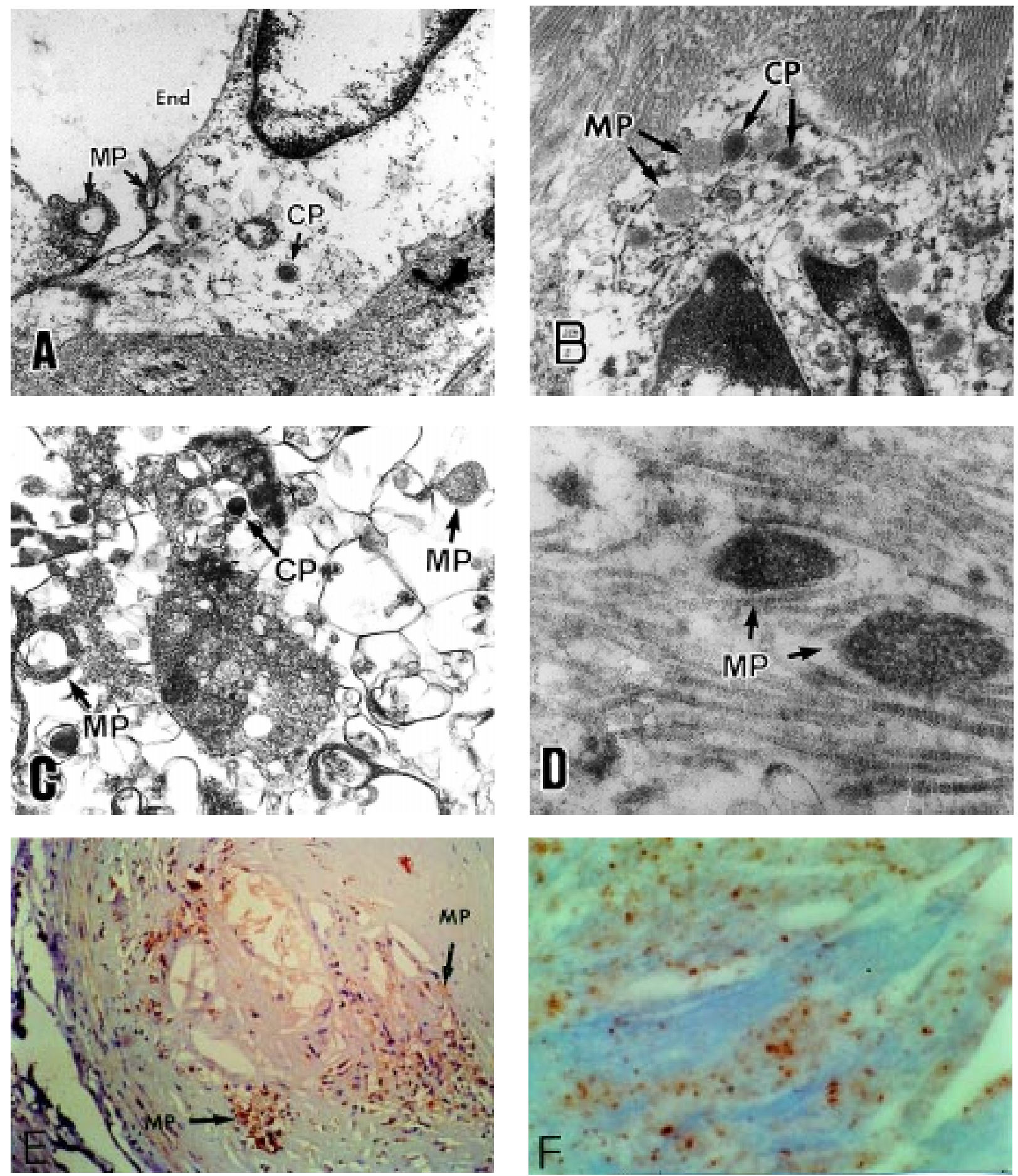

Figure 1 - Electron microscopy (A-D) and histopathological view of in situ hybridization with an M. pneumoniae bioprobe $(E, F)$ of fatal ruptured plaque coronary artery segments. A, Endothelial cell (End) of vasa vasorum exhibiting very small forms of Mycoplasma pneumoniae (MP) adhered to the endothelial surface. Presence of an elementary body of Chlamydia pneumoniae (CP) in the cytoplasm. (original magnification: 3,300X). B, Adventitial macrophage containing several CP bodies and MP forms (2,600X). C, Necrotic core of atheroma plaque exhibiting many CP bodies and MP forms among abundant ruptured membrane elements $(4,200 X)$. D, Two ellipsoid forms of MP in the interstitium (10,000X). E, Several positive rounded brownish structures of MP inside a vulnerable plaque $(1,000 \mathrm{X})$. F, Closer view of a necrotic atheromatous core exhibiting many positive brownish dots corresponding to MP $(1,000 \mathrm{X})$. 


\section{References}

1. Boyle JJ (1997). Association of coronary plaque rupture and atherosclerotic inflammation. J oumal of Pathology, 181: 93-99.

2. Shor A \& Phillips JI (1999). Chlamydia pneumoniae and atherosclerosis. J ournal of the American Medical Association, 282: 2071-2073.

3. de Boer OJ , van der Wal AC \& Becker AR (2000). Atherosclerosis, inflammation and infection. J ournal of Pathology, 190: 237243.

4. Higuchi ML, Castelli JB, Aiello VD, Palomino $S$, Reis MM, Sambiase NV, Fukasawa S, Bezerra HG \& Ramires JA (2000). Great amount of C. pneumoniae in ruptured plaque vessel segments at autopsy. A comparative study of stable plaques. Arquivos Brasileiros de Cardiologia, 74: 149-151.

5. Razin S, Yogev D \& Naot Y (1998). Molecular biology and pathogenicity of mycoplasmas. Microbiology and Molecular Biology Reviews, 62: 1094-1156.

6. Sambiase NV, Higuchi ML, Nuovo G, Gutierrez P, Fiorelli Al, Uip DE \& Ramires J A (2000). CMV and transplant-related cor- onary atherosclerosis: an immunohistochemical, in situ hybridization and polymerase chain reaction in situ study. Modem Pathology, 13: 173-179.

7. Almagor M, Kahane I \& Yatziv S (1984). Role of superoxide anion in host cell injury induced by M. pneumoniae infection. A study in normal and trisomy 21 cells. J ournal of Clinical Investigation, 73: 842847.

8. Stacey A \& Bradlow A (1999). Arcanobacterium haemolyticum and Mycoplasma pneumoniae co-infection. J ournal of Infection, 38: 41-42.

9. Meijer A, van der Vliet JAQ, Roholl PJ, Gielis-Proper SK, de Vries A \& Ossewaarde J M (1999). Chlamydia pneumoniae in abdominal aortic aneurysms: abundance of membrane components in the absence of heat shock protein 60 and DNA. Arteriosclerosis, Thrombosis, and Vascular Biology, 19: 2680-2686.

10. Blasi F, Fagetti L \& Allegra L (2000). Chlamydia pneumoniae detection in atherosclerotic plaques in Italy. J ournal of Infectious Diseases, 181 (Suppl 3): S444-S446.
11. Corsaro $D$, Valassina $M$, Venditti $D$, Venard V, Faou AL \& Valensin PE (1999). Multiplex PCR for rapid and differential diagnosis of Mycoplasma pneumoniae and Chlamydia pneumoniae in respiratory infections. Diagnostic Microbiology and Infectious Disease, 35: 105-108.

12. Tong CYW, Donnelly C, Harvey $G \&$ Sillis M (1999). Multiple polymerase chain reaction for the simultaneous detection of Mycoplasma pneumoniae, Chlamydia pneumoniae and Chlamydia psittaci in respiratory samples. J oumal of Clinical Pathology, 52: 257-263.

13. Horne BD, Muhlestein J B, Carlsquist J F, Ohana B, Lipson M, Kahn N, Habashi J, Bair T \& Anderson J L (2000). IgA seropositivity to Mycoplasma pneumoniae predicts the diagnosis of coronary artery disease. J oumal of the American College of Cardiology, 35: 321 (Abstract).

14. Fong IW, Chiu B, Viira E, J ang D \& Mahony J B (1999). De novo induction of atherosclerosis by $\mathrm{C}$. pneumoniae in a rabbit model. Infection and Immunity, 67: 6048-6055. 